\title{
A Comparison of Thin-plate Splines with Automatic Correspondences and B-splines with Uniform Grids for Multimodal Prostate Registration
}

\author{
Jhimli Mitra ${ }^{a, b}$, Robert Martí ${ }^{b}$, Arnau Oliver $^{b}$, Xavier Lladó $^{b}$, Joan C. Vilanova $^{c}$ \\ and Fabrice Meriaudeau ${ }^{a}$ \\ ${ }^{a}$ Université de Bourgogne, Le2i-UMR CNRS 5158, Le Creusot, France. \\ ${ }^{b}$ Universitat de Girona, Computer Vision and Robotics Group, Girona, Spain. \\ ${ }^{c}$ Clínica Girona, Girona, Spain.
}

\begin{abstract}
This paper provides a comparison of spline-based registration methods applied to register interventional Trans Rectal Ultrasound (TRUS) and pre-acquired Magnetic Resonance (MR) prostate images for needle guided prostate biopsy. B-splines and Thin-plate Splines (TPS) are the most prevalent spline-based approaches to achieve deformable registration. Pertaining to the strategic selection of correspondences for the TPS registration, we use an automatic method already proposed in our previous work to generate correspondences in the MR and US prostate images. The method exploits the prostate geometry with the principal components of the segmented prostate as the underlying framework and involves a triangulation approach. The correspondences are generated with successive refinements and Normalized Mutual Information (NMI) is employed to determine the optimal number of correspondences required to achieve TPS registration. B-spline registration with successive grid refinements are consecutively applied for a significant comparison of the impact of the strategically chosen correspondences on the TPS registration against the uniform B-spline control grids. The experimental results are validated on 4 patient datasets. Dice Similarity Coefficient (DSC) is used as a measure of the registration accuracy. Average DSC values of $0.97 \pm 0.01$ and $0.95 \pm 0.03$ are achieved for the TPS and B-spline registrations respectively. B-spline registration is observed to be more computationally expensive than the TPS registration with average execution times of $128.09 \pm 21.70$ seconds and $62.83 \pm 32.77$ seconds respectively for images with maximum width of 264 pixels and a maximum height of 211 pixels.
\end{abstract}

Keywords: Prostate biopsy, multimodal registration, thin-plate splines, B-splines, automatic correspondences.

\section{INTRODUCTION}

Countries in Europe and USA have been following prostate cancer screening programs since last 15 years. A patient with abnormal findings after a digital rectal examination, serum Prostate Specific Antigen (PSA) level over $4.0 \mathrm{ng} / \mathrm{ml}$ and PSA velocity more than 0.4 to $0.75 \mathrm{ng} / \mathrm{ml} / \mathrm{yr}$ is generally advised with a prostate biopsy for histopathological examination of the prostate tissues to diagnose benign or malignant lesions. The most common appearance of malignant lesions in a TRUS guided needle biopsy is hypoechoic. The accuracy of sonographic finding of hypoechoic prostate cancer lesions is typically $43 \%{ }^{1}$ The prevalence of isoechoic prostate cancer lesions on TRUS ranges from $25 \%-42 \%$. To uncover these isoechoic lesions, urologists adopt a multi core

Further author information: (Send correspondence to Jhimli Mitra or Dr. Robert Martí or Prof. Fabrice Meriaudeau.) Jhimli Mitra: E-mail: jhimlimitra@yahoo.com,

Robert Martí: E-mail: marly@eia.udg.edu, Telephone: +34972418876

Arnau Oliver: E-mail: aoliver@eia.udg.edu,

Xavier Lladó: E-mail: llado@eia.udg.edu,

Joan C. Vilanova: E-mail: kvilanova@comg.cat,

Fabrice Meriaudeau: E-mail: fabrice.meriaudeau@u-bourgogne.fr, Telephone: +33(0)385731077

Medical Imaging 2011: Visualization, Image-Guided Procedures, and Modeling,

edited by Kenneth H. Wong, David R. Holmes III, Proc. of SPIE Vol. 7964, 79642T

(C) 2011 SPIE - CCC code: 0277-786X/11/\$18 - doi: 10.1117/12.877956

Proc. of SPIE Vol. 7964 79642T-1 
biopsy technique and strategically extract 6-11 biopsy samples. However, the multi core biopsy technique often fails to uncover the malignant lesions and the number of re-biopsies increase. Intending to solve this problem, a registration method is employed to fuse the TRUS image with a preacquired corresponding MR prostate image resliced from a MR volume. ${ }^{2-5}$

A deformable registration method needs to be applied to accommodate the deformations of the prostate in the respective modalities due to different patient positions on the couch, full bladder, bowel and gas in rectum, insertion of the endorectal probe and inflation of the endorectal balloon inside the rectum during MRI. ${ }^{6}$ In this work, the possibilities of using B-splines or TPS for deformable registration with their accuracies and complexities involved during interventional biopsy procedures are being explored. An existing method to generate correspondences are used for TPS registration. ${ }^{7}$ The results show that the TPS registration method fuses the TRUS and MR prostate images efficiently and accurately in case of large deformations than the traditional B-spline method with uniform control points .

The remaining of the paper is organized in the following manner. Section 2 provides a short description of the method of correspondences and the TPS registration that uses such correspondences. Section 3 describes the B-spline registration method. Section 4 provides the results and discussions followed by conclusions in section 5 .

\section{THIN-PLATE SPLINES REGISTRATION}

The first part of this section briefly describes the previously established correspondence method followed by a detailed explanation of TPS formulation in the second part.

\subsection{Automatic Correspondences}

In our work, the prostate regions are segmented manually from the corresponding MR and Ultra Sound (US) images. However, we are also investigating on automatic prostate segmentation. ${ }^{8}$ The US image is treated as the reference and the MR as the moving image. The TPS registration involves a set of correspondences generated by a geometric method based on the geometry of the segmented prostate contours in the respective modalities. The principal axes of the prostate obtained from PCA of the contour are used as the underlying framework for our algorithm. The US image principal axes are projected and centered on the MR prostate contour.

The method of generating correspondences is based on a triangulation approach in different levels. Let $p_{i}$, where, $i=1, \ldots, n, n=4$ for level $r=0$, represent the the four intersections of the principal axes with the prostate contour. With the final level $R$, the algorithm is as follows:

1. Level $r=1$.

2. Loop while $r<=R$.

3. Generate midpoint $q_{i}$ between $p_{i}$ and $p_{i+1}$ as $\left(p_{i}+p_{i+1}\right) / 2$.

4. Find a point $x_{i}$ on the contour between $p_{i}$ and $p_{i+1}$ such that $\operatorname{slope}\left(p_{i}, p_{i+1}\right) \cdot \operatorname{slope}\left(x_{i}, q_{i}\right)=-1$.

5. $\left(p_{i}, x_{i}, p_{i+1}\right)$ comprise the triangulated region of the prostate between $p_{i}$ and $p_{i+1}$.

6. Repeat Steps 3-5 until $p_{i}=p_{n}$ and $p_{i+1}=p_{1}$.

7. If $r<=R$, then update $n=2 n$ and $r=r+1$, save $p_{1}, x_{1}, p_{2}, \ldots, p_{n-1}, x_{n-1}, p_{n}, x_{n}$ as new $p_{i}$ s with $i=1, \ldots, n$ and repeat from Step 3. Else, end the loop.

Fig. 1 shows the triangulation method for three subsequent levels and the obtained correspondence points. For accurate deformation certain correspondences are also generated inside the prostate contour that are the $q_{i}$ of level $r=1$ (see Fig. 1(b)).

After each level of correspondences being generated, a TPS registration is performed and the resulting NMI is computed to measure the similarity between the transformed moving image and the reference image. The level at which the maximum NMI is obtained is considered as the optimal level of correspondences. A detailed description of the method is available in Mitra et al. ${ }^{7}$

Proc. of SPIE Vol. 7964 79642T-2 


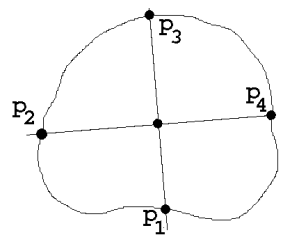

(a) Level 0

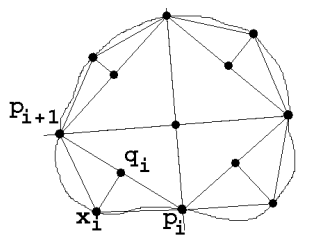

(b) Level 1

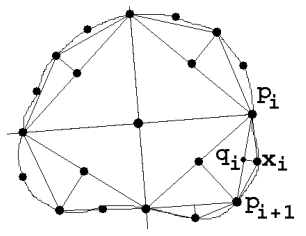

(c) Level 2

Figure 1. Method of generating correspondence points in different levels.

\subsection{Formulation of TPS}

The thin-plate spline is a commonly used basis function in $2 \mathrm{D}$-Euclidean space ${ }^{9}$ to map the coordinates of a moving image into a reference image, when a set of homologous correspondence points are established in both images. In its extended form, the deformable TPS model includes the affine model as a special case.

If $p_{i}=\left(x_{i}, y_{i}\right)$ and $q_{i}=\left(x_{j}, y_{j}\right), i=j=1, \ldots, n$ represent two sets of corresponding landmarks in the moving and reference images respectively, then, the TPS interpolation $f(x, y)$ minimizes the bending energy

$$
I_{f}=\iint_{\Re^{2}}\left(f_{x x}^{2}+2 f_{x y}^{2}+f_{y y}^{2}\right) d x d y
$$

and has the form

$$
\begin{aligned}
& f(x, y)=a_{1}+a_{x} x+a_{y} y+ \\
& \sum_{i=1}^{n} w_{i} U\left(\left\|\left(x_{i}, y_{i}\right)-(x, y)\right\|\right)
\end{aligned}
$$

where, $U(r)=r^{2} \log r, a_{1}, a_{x}$ and $a_{y}$ are the affine parameters and $w_{i}$ s are the TPS parameters and $f(x, y)$ should have square integrable derivatives if

$$
\sum_{i=1}^{n} w_{i}=0 \quad \text { and } \quad \sum_{i=1}^{n} w_{i} x_{i}=\sum_{i=1}^{n} w_{i} y_{i}=0 .
$$

The boundary conditions yields a linear system of equation for the TPS coefficients and could be solved analytically as

$$
\left[\begin{array}{cc}
K & P \\
P^{T} & O
\end{array}\right]\left[\begin{array}{l}
w \\
a
\end{array}\right]=\left[\begin{array}{l}
q \\
o
\end{array}\right]
$$

where, $K$ is a $n \times n$ matrix and $K_{i j}=U\left(\left\|\left(x_{i}, y_{i}\right)-\left(x_{j}, y_{j}\right)\right\|\right)$, ith row of $P$ is $\left(1, x_{i}, y_{i}\right), O$ is a $3 \times 3$ matrix of zeros, $o$ is a $3 \times 1$ column vector of zeros, $w$ and $q$ are column vectors of $w_{i}$ s and $q_{j}$ s respectively, $a$ is a column vector of the affine parameters $a_{1}, a_{x}$ and $a_{y}$.

Localization errors of the correspondence points may be considered by extending the interpolation to regularization. ${ }^{10}$ This is accomplished by the minimization of

$$
H(f)=\sum_{i=1}^{n} \frac{\left(q_{i}-f\left(x_{i}, y_{i}\right)\right)^{2}}{\sigma_{i}^{2}}+\lambda I_{f}
$$


The covariance $\sigma_{i}^{2}$ is the sum of the covariances of the points $p_{i}$ and $q_{i}$ and $\lambda=0.01$ is the regularization term. Therefore, the TPS linear system of equations in (3) may be rewritten as

$$
\left[\begin{array}{cc}
K+n \lambda C^{-1} & P \\
P^{T} & O
\end{array}\right]\left[\begin{array}{l}
w \\
a
\end{array}\right]=\left[\begin{array}{l}
q \\
o
\end{array}\right]
$$

where,

$$
C^{-1}=\left(\begin{array}{ccc}
\sigma_{1}^{2} & & 0 \\
& \ddots & \\
0 & & \sigma_{n}^{2}
\end{array}\right)
$$

Introducing the term $n \lambda C^{-1}$ yields a better conditioned linear system and a robust numerical solution. Finally, (4) is framed as

$$
L U=V
$$

and solved as

$$
U=L^{-1} V
$$

where,

$$
L=\left[\begin{array}{cc}
K+n \lambda C^{-1} & P \\
P^{T} & O
\end{array}\right], U=\left[\begin{array}{c}
w \\
a
\end{array}\right]
$$

and

$$
V=\left[\begin{array}{l}
q \\
o
\end{array}\right]
$$

\section{B-SPLINES REGISTRATION}

A common technique to represent a free-form deformation is to employ spline functions as B-splines. ${ }^{11}$ B-splines consist of set of control points that can be locally controlled on the image domain. Although, the original Bsplines equation is formulated for $3 \mathrm{D}$-deformable objects, ${ }^{11,12}$ the spline functions in this paper are represented for $2 \mathrm{D}$ images.

Let $\Omega=\{(x, y) \mid 0 \leq x<X, 0 \leq y<Y\}$ represent the image domain. The transformation between the floating and reference images is given by $\mathbf{T}:(x, y) \mapsto\left(x^{\prime}, y^{\prime}\right)$, where any point $(x, y)$ of the floating image is mapped onto its corresponding point $\left(x^{\prime}, y^{\prime}\right)$ on the reference image. Given a mesh of control points $(\Phi)$ on the floating image as $\phi_{i, j}$ with uniform spacing of $\delta \mathrm{mm}$, the nonrigid transformation $\mathbf{T}$ is defined by B-spline functions as

$$
\mathbf{T}(x, y)=\sum_{l=0}^{3} \sum_{m=0}^{3} B_{l}(u) B_{m}(v) \phi_{i+l, j+m}
$$

where $i=\lfloor x / \delta\rfloor-1, j=\lfloor y / \delta\rfloor-1, u=x / \delta-\lfloor x / \delta\rfloor$ and $v=y / \delta-\lfloor y / \delta\rfloor . B_{l}$ represents the $l^{t h}$ basis function of the cubic B-spline functions such that

$$
\begin{aligned}
& B_{0}(u)=\left(1-u^{3}\right) / 6 \\
& B_{1}(u)=\left(3 u^{3}-6 u^{2}+4\right) / 6 \\
& B_{2}(u)=\left(-3 u^{3}+3 u^{2}+3 u+1\right) / 6 \\
& B_{3}(u)=u^{3} / 6
\end{aligned}
$$


The B-spline free-form deformations are locally controlled because the deformation at any point $(x, y)$ is controlled by its neighboring $4 \times 4$ control points. B-splines provide a wide range of deformations by organizing the mesh of control points and the images in a hierarchy, ${ }^{13}$ i.e. the distance between the control points increase introducing more control points while the images move from coarser to finer levels. The B-spline control points grid refinement is done using the standard splitting matrix. ${ }^{14}$

The similarity measure used for B-splines deformation is NMI between the moving $(M)$ and the reference $(R)$ images $^{15}$ and is given by

$$
\mathrm{NMI}=\zeta_{\text {similarity }}=\frac{H(M)+H(R)}{H(M, R)}
$$

where $H(M)$ and $H(R)$ are the marginal entropies of the moving and reference images respectively, and $H(M, R)$ is the joint entropy of the images. Therefore, the cost function for optimization is defined as

$$
\zeta(\Phi)=-\zeta_{\text {similarity }}(R, \mathbf{T}(M)) .
$$

The optimization is solved using a quasi-Newton optimization method as "Limited Memory Broyden-FletcherGlodfarb-Shanno" (L-BFGS) algorithm. ${ }^{16}$

\section{RESULTS AND DISCUSSIONS}

Datasets of 4 patients are used to evaluate the results of B-spline with uniform control grids and TPS registration with our method of correspondences. The axial middle slices of US/MR are primarily used in this experiment. The TRUS images are acquired by SIEMENS ACUSON and the T2-weighted MR volumes by GE Signa HDx 1.5 Tesla machines. B-spline registration with initial uniform grid spacing of $64 \times 64$ pixels is implemented. Grid refinements are done up to 2 levels over the initial control grid to improve the accuracy of registration. Similarly, TPS registration is implemented on the basis of correspondences generated at each level from 1 to 3 . For a meaningful comparison both the registration methods involve control grid or correspondence refinements up to 3 levels, with an exception that the moving image is always transformed with the TPS parameters after each level of correspondences are being generated.

Table 1 shows the quantitative comparison of B-spline and TPS in terms of optimal correspondence points, control grids, time and DSC values. The initial grid number depends on uniform $64 \times 64$ pixel spacing and the image size. It is observed from our experiments that the best registration results in terms of the resulting DSC values are always obtained with level 3 control grid for B-splines. However, as shown in Table 1, accurate registration results are obtained also with varied levels of correspondences for the TPS interpolation. It is observed from Table 1 that B-spline requires more time than TPS registration for each patient. This is due to the optimizer involved with B-spline that tries to maximize the NMI with increased number of control grids. In contrast, TPS framework involves a linear system of equations that can be solved easily with least-squares method and works accurately with lesser correspondences. Patient 3 shows a slight increase in DSC value with B-spline than TPS at the cost of more control points and time. Table 2 shows the NMI values for each patient to determine the optimal level of correspondences, i.e. the column corresponding to the maximum NMI value for a patient.

Table 1. Quantitative comparison of B-spline and TPS registration

\begin{tabular}{|c|c|c|c|c|c|c|c|c|}
\hline \multirow{2}{*}{ Patient } & \multicolumn{4}{|c|}{ B-splines } & \multicolumn{3}{c|}{ TPS } \\
\cline { 2 - 9 } & Intial Grid & Level 2 Grid & Level 3 Grid & Time (secs) & DSC & Correspondences & Time (secs) & DSC \\
\hline 1 & $7 \times 7$ & $10 \times 11$ & $17 \times 19$ & $140.47 \pm 5.52$ & 0.98 & Level 3/37 points & 94.78 & 0.98 \\
\hline 2 & $6 \times 8$ & $9 \times 12$ & $15 \times 20$ & $126.30 \pm 9.17$ & 0.97 & Level 2/21 points & 84.11 & 0.97 \\
\hline 3 & $6 \times 6$ & $8 \times 9$ & $13 \times 15$ & $98.29 \pm 2.31$ & 0.98 & Level 2/21 points & 22.85 & 0.96 \\
\hline 4 & $6 \times 8$ & $8 \times 12$ & $12 \times 20$ & $147.31 \pm 0.36$ & 0.90 & Level 3/37 points & 50.31 & 0.97 \\
\hline
\end{tabular}


Table 2. NMI after TPS registration with correspondences at different levels

\begin{tabular}{|c|c|c|c|}
\hline Patient & Level 1 & Level 2 & Level 3 \\
\hline 1 & -260.24 & -174.23 & $\mathbf{- 1 6 7 . 6 7}$ \\
\hline 2 & -781.48 & $\mathbf{- 4 1 2 . 0 1}$ & -424.14 \\
\hline 3 & -419.79 & $\mathbf{- 4 0 8 . 5 4}$ & -408.68 \\
\hline 4 & -454.64 & -403.03 & $\mathbf{- 3 8 9 . 4 5}$ \\
\hline
\end{tabular}

Fig. 2 shows the registration results with TPS from optimal correspondences and B-spline with level 3 refinement of control grid. We observe that TPS and B-spline provide qualitatively similar results for patients 1 , 2 and 3, while B-spline registration completely fails for patient 4. It is to be noted that the B-spline registration involves uniform control grid placed over the MR images. We have observed from our experiments that refinement of control grid after level 3 does not improve the B-spline results and sometimes deteriorated results are obtained both with B-splines and TPS with the current resolution of the images. Fig. 3 shows the TPS registration qualities for patient 4 at different levels of correspondences that are in conformity with the NMI values of Table 2. All the implementations are done in MATLAB R2009b with Core2Duo 1.66 GHz processor and 2GB memory.

\section{CONCLUSIONS AND FUTURE WORKS}

TPS registration using point correspondences automatically generated on interventional TRUS and preacquired MR prostate images has been proposed. Significant comparisons in terms of registration accuracy and efficiency between commonly used spline-based registration techniques like B-spline with uniform control grids and TPS with our previously established method of correspondences have been presented. It has been observed that TPS performs more accurate and time efficient registrations than B-splines. NMI used to determine the optimal level of correspondences used for the TPS registration always provided high values for the optimal level for all the analyzed cases.

In the future, we propose to extend our method of generating correspondences for registration of 3D volumes. The correspondence generation method with TPS would be of practical significance for slice to volume registration during TRUS interventional biopsy with the parallelization of the processes at different levels using a GPU.

\section{ACKNOWLEDGMENTS}

This research is a part of the PROSCAN project of the VICOROB laboratory of University of Girona, Catalunya, Spain. The authors would like to thank VALTEC 08-1-0039 of Generalitat de Catalunya, Spain and Conseil Régional de Bourgogne, France for funding this research.

\section{REFERENCES}

[1] Carroll, P. and Shinohara, K., "Transrectal ultrasound guided prostate biopsy," tech. rep., Department of Urology, University of California, San Francisco (2010). http://urology.ucsf.edu/patientGuides/pdf/uroOnc/Prostate_Biopsy.pdf, accessed [18th Oct, 2010].

[2] Kaplan, I., Oldenburg, N. E., Meskell, P., Blake, M., Church, P., and Holupka, E. J., "Real time MRIultrasound image guided stereotactic prostate biopsy," Magnetic Resonance Imaging 20, 295-299 (2002).

[3] Reynier, C., Troccaz, J., Fourneret, P., Dusserre, A., Gay-Jeune, C., Descotes, J.-L., Bolla, M., and Giraud, J.-Y., "MRI/TRUS data fusion for prostate brachytherapy. preliminary results," Medical Physics 31(6), 1568-1575 (2004).

[4] Singh, A. K., Kruecker, J., Xu, S., Glossop, N., Guion, P., Ullman, K., Choyke, P. L., and Wood, B. J., "Initial clinical experience with real-time transrectal ultrasonography-magnetic resonance imaging fusionguided prostate biopsy," British Journal of Urology International 101(7), 841-845 (2008).

[5] Xu, S., Kruecker, J., Turkbey, B., Glossop, N., Singh, A. K., Choyke, P., Pinto, P., and Wood, B. J., "Real-time MRI-TRUS fusion for guidance of targeted prostate biopsies," Computer Aided Surgery 13(5), $255-264$ (2008). 
[6] Daanen, V., Gastaldo, J., Giraud, J. Y., Fourneret, P., Descotes, J. L., Bolla, M., Collomb, D., and Troccaz, J., "MRI/TRUS data fusion for brachytherapy," Intl. Journal of Medical Robotics and Computer Assisted Surgery 2(3), 256-261 (2006).

[7] Mitra, J., Oliver, A., Martí, R., Lladó, X., Vilanova, J. C., and Meriaudeau, F., "A thin-plate spline based multimodal prostate registration with optimal correspondences." Sixth International Conference on Signal-Image Technology and Internet-Based Systems (SITIS'10) (2010).

[8] Ghose, S., Oliver, A., Martí, R., Lladó, X., Freixenet, J., Vilanova, J., and Meriaudeau, F., "Texture guided Active Appearance Model propagation for prostate segmentation," MICCAI Workshop on Prostate Cancer Imaging, Computer Aided Diagnosis, Prognosis and Intervention, LNCS 6367, 111-120 (2010).

[9] Bookstein, F. L., [Morphometric Tools for Landmark Data: Geometry and Biology], Cambridge University Press, Cambridge, UK (1991).

[10] Rohr, K., Stiehl, H. S., Sprengel, R., Buzug, T. M., Weese, J., and Kuhn, M. H., "Landmark-based elastic registration using approximating thin-plate splines," IEEE Transactions on Pattern Analysis and Machine Intelligence 20(6), 526-534 (2001).

[11] Rueckert, D., Sonoda, L. I., Hayes, C., Hill, D. L. G., Leach, M. O., and Hawkes, D. J., "Nonrigid registration using free-form deformations: Application to breast MR images," IEEE Transactions on Medical Imaging 18(8), 712-721 (1999).

[12] Ino, F., Ooyama, K., and Hagihara, K., "A data distributed parallel algorithm for nonrigid image registration," Parallel Computing 31, 19-43 (2005).

[13] Forsey, D. R. and Barrels, R. H., "Hierarchcal B-spline refinement," Computer Graphics 22(4), 205-212 (1988).

[14] Yamaguchi, F., [Curves and Surfaces in Computer Aided Geometric Design], Springer (1988).

[15] Studholme, C., Hill, D. L. G., and Hawkes, D. J., "An overlap invariant entropy measure of 3D medical image alignment," Pattern Recognition 72(1), 71-86 (1999).

[16] Liu, D. C. and Nocedal, J., "On the limited memory method for large scale optimization," Mathematical Programming B 45(3), 503-528 (1989). 

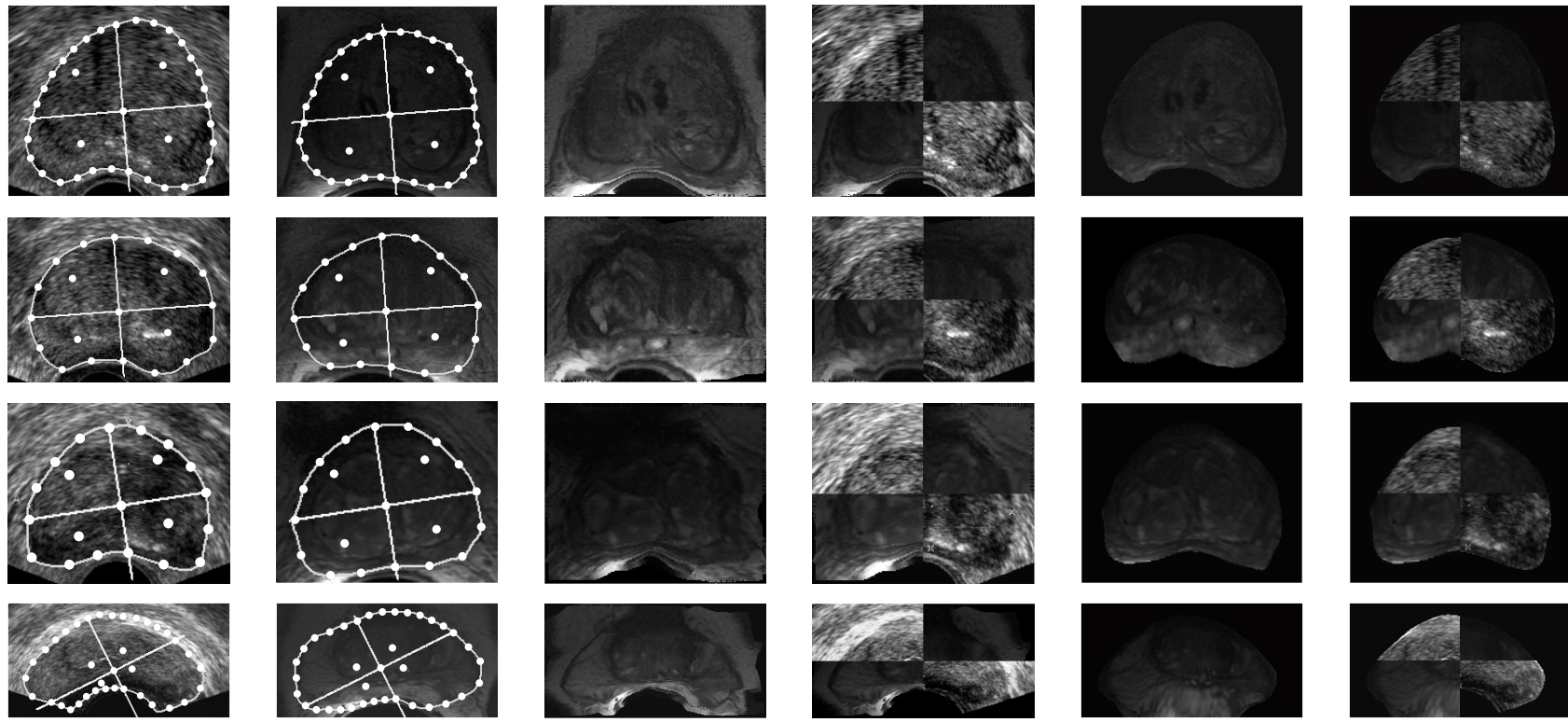

Figure 2. Registration results with TPS and B-splines for patients 1-4 in rows. (columns 1 and 2) US and MR images with optimal correspondences, (column 3) transformed MR image with TPS, (column 4) checker board for TPS registration, (column 5) transformed MR image with B-splines Level 3 grid, (column 6) checkerboard for B-spline registration
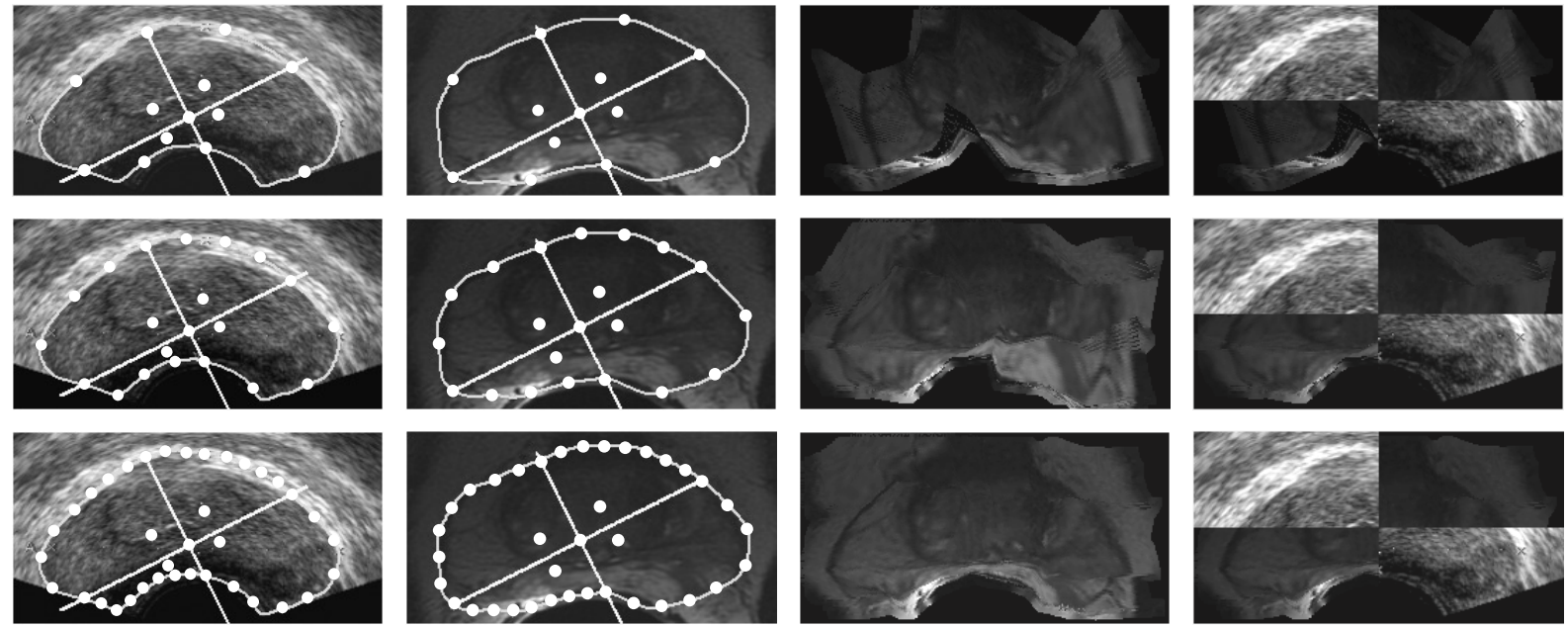

Figure 3. Patient-4 quality of registrations for different levels of correspondences using our method. (First-row) Level 1 correspondences, (second-row) level 2 correspondences, (third-row) level 3 correspondences. The column representations are the same as Fig. 2. 